\title{
Trauma esplénico: diagnostico, clasificación y tratamiento. Una revisión de la literatura actual
}

\section{Splenic trauma: diagnosis, classification and treatment. A review of the current literature}

Trauma esplênico: diagnóstico, classificação e tratamento. Uma revisão da literatura atual

\author{
Sebastián Alejandro Pástor Romero \\ sebastian.pastor.md@gmail.com \\ https://orcid.org/0000-0001-9401-0389
}

\author{
Michelle Nicole Maldonado Brito \\ mnmaldonado@unach.edu.ec \\ https://orcid.org/0000-0003-3069-3541
}

\author{
Erika Paulina Monar Naranjo \\ epmonar@unach.edu.ec \\ https://orcid.org/0000-0001-9791-1166
}

\author{
Walter Omar Villacres Salazar \\ wovillacres.fsm@unach.edu.ec \\ https://orcid.org/0000-0002-3104-5039
}

Paola Alexandra Medina Flores
paola.medina.md@gmail.com
https://orcid.org/0000-0001-5796-4714

Cynthia Vanessa Díaz Barahona

cvdiaz@unach.edu.ec

https://orcid.org/0000-0002-8822-9891

\section{Hospital General Riobamba, Instituto Ecuatoriano de Seguridad Social Universidad Nacional de Chimborazo, Riobamaba-Ecuador}

Recibido 29 de marzo 2021 | Arbitrado y aceptado 16 de abril 2021 | Publicado en 16 de agosto 2021

\begin{abstract}
RESUMEN
El bazo es el órgano que se lesiona con más frecuencia en el trauma abdominal cerrado, presentándose en 30 a $50 \%$ de los casos, principalmente por su fragilidad y localización, su tratamiento ha estado en constante cambio a lo largo del tiempo, siendo hoy en día más utilizado el manejo conservador por sobre el intervencionista, considerando sobre todo el mayor riesgo de mortalidad y las condiciones fisiológicas posteriores a la esplenectomía principalmente en niños y adolescentes. Objetivo. Realizar una revisión actual del diagnóstico, clasificación y tratamiento del trauma esplénico. Metodología. Se realizó una revisión bibliográfica incluyendo los descriptores relacionados con trauma esplénico y su tratamiento. Resultados. La identificación del trauma esplénico es de vital importancia para la supervivencia del paciente, el diagnostico se puede realizar mediante estudios de imagen eco-FAST o tomografía en dependencia del estado hemodinámico del paciente, la clasificación depende de las características anatómicas de las lesiones y orienta el tratamiento adecuado. Conclusiones. Actualmente el tratamiento conservador está recomendado para lesiones I-III; los estadios mayores (IV y V) o cualquier grado siempre y cuando exista compromiso hemodinámico implican tratamiento intervencionista o quirúrgico, no existe una diferencia significativa entre la utilización de técnica abierta vs laparoscópica.
\end{abstract}

Palabras clave: Trauma abdominal cerrado; trauma esplénico; bazo; tratamiento

\begin{abstract}
The spleen is the organ that is most frequently injured in blunt abdominal trauma, occurring in 30 to $50 \%$ of cases, mainly due to its fragility and location, its treatment has been in constant change over time, today the conservative management is more widely used than interventionist, considering above all the greater risk of mortality and the physiological conditions after splenectomy, mainly in children and adolescents. Objective. To carry out a current review of the diagnosis, classification and treatment of splenic trauma. Methodology. A bibliographic review was carried out including the descriptors related to splenic trauma and its treatment. Results. The identification of splenic trauma is of vital importance for the survival of the patient, the diagnosis can be made by imaging studies, FAST ultrasound or tomography depending on the hemodynamic status of the patient, the classification depends on the anatomical characteristics of the lesions and guides the appropriate treatment. Conclusions. Conservative treatment is currently recommended for lesions I-III; the major stages (IV and V) or any grade whit hemodynamic compromise imply interventional or surgical treatment, there is no significant difference between the use of open versus laparoscopic technique.
\end{abstract}

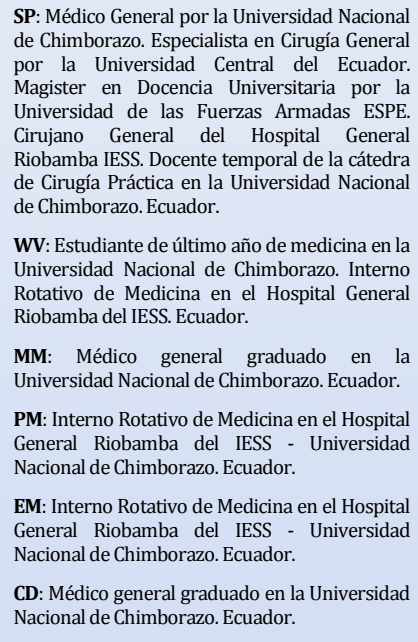
SP: Médico General por la Universidad Nacional
de Chimborazo. Especialista en Cirugía General por la Universidad Central del Ecuador. Magister en Docencia Universitaria por la Magister en Docencia Universitaria por la
Universidad de las Fuerzas Armadas ESPE. Cirujano General del Hospital General Riobamba IESS. Docente temporal de la cátedra de Cirugía Práctica en la Universidad Nacional de Cirugía Práctica en la
de Chimborazo. Ecuador.

WV: Estudiante de último año de medicina en la Universidad Nacional de Chimborazo. Interno Riobamba del IESS. Ecuador.

MM: Médico general graduado en la Universidad Nacional de Chimborazo. Ecuador. PM: Interno Rotativo de Medicina en el Hospital General Riobamba del IESS - Universidad Nacional de Chimborazo. Ecuador.

EM: Interno Rotativo de Medicina en el Hospital General Riobamba del IESS - Universidad Nacional de Chimborazo. Ecuador.

CD: Médico general graduado en la Universidad Nacional de Chimborazo. Ecuador.

Key words: Blunt abdominal trauma; splenic trauma; spleen; treatment 
SP: Médico General por la Universidad Naciona de Chimborazo. Especialista en Cirugía Genera por la Universidad Central del Eciador. Magister en Docencia Universitaria por la Universida de las Cinuano Genel del Hospit Genera Cirujano General del Hospital General Riobamba IESS. Docente temporal de la cátedra de Cirugía Práctica en la Universidad Nacional de Chimborazo. Ecuador.

WV: Estudiante de último año de medicina en la Universidad Nacional de Chimborazo. Interno Rotativo de Medicina en el Hospital General Riobamba del IESS. Ecuador.

MM. Médico general graduado en la

PM: Interno Rotativo de Medicina en el Hospital General Riobamba del IESS - Universidad Nacional de Chimborazo. Ecuador.

EM: Interno Rotativo de Medicina en el Hospital General Riobamba del IESS - Universidad Nacional de Chimborazo. Ecuador.

CD: Médico general graduado en la Universidad Nacional de Chimborazo. Ecuador.

\begin{abstract}
RESUMO
0 baço é o órgão mais frequentemente lesado no trauma abdominal fechado, ocorrendo em 30 a $50 \%$ dos casos, principalmente devido à sua fragilidade e localização, seu tratamento tem mudado constantemente ao longo do tempo, sendo hoje o manejo conservador mais utilizado que o intervencionista, considerando sobretudo o maior risco de mortalidade e as condições fisiológicas após a esplenectomia, principalmente em crianças e adolescentes. Objetivo. Fazer uma revisão atual do diagnóstico, classificação e tratamento do trauma esplênico. Metodologia. Foi realizada uma revisão bibliográfica incluindo os descritores relacionados ao trauma esplênico e seu tratamento. Resultados. A identificação do trauma esplênico é de vital importância para a sobrevida do paciente, o diagnóstico pode ser feito por exames de imagem echo-FAST ou tomografia dependendo do estado hemodinâmico do paciente, a classificação depende das características anatômicas das lesões e orienta o tratamento adequado. Conclusões. 0 tratamento conservador é atualmente recomendado para lesões I-III; os estágios principais (IV e V) ou em qualquer grau, desde que haja comprometimento hemodinâmico, implicam em tratamento intervencionista ou cirúrgico, não havendo diferença significativa entre o uso da técnica aberta e laparoscópica.
\end{abstract}

Palavras-Chave: Trauma abdominal fechado, trauma esplênico, baço, tratamento

\section{INTRODUCCIÓN}

$\mathrm{E}$ l bazo es un órgano encapsulado y considerablemente frágil, lo cual hace que se encuentre comprometido en el trauma abdominal cerrado con mayor frecuencia, con un daño que corresponde de 3 a $5 \%$ del trauma abdominal grave en el adulto y menos del $1 \%$ en pacientes pediátricos, siendo en este último grupo etario donde ocurren repercusiones graves si su daño es total y por lo tanto aquí radica la importancia del correcto manejo del trauma esplénico y la actualización de conocimientos, buscando reducir considerablemente las consecuencias a futuro.

Es bien conocido que, durante los años 50 , la manera de reducir la mortalidad por trauma esplénico fue la esplenectomía como primera opción, teniendo resultados a corto plazo relativamente alentadores. Sin embargo, a lo largo de los años, el manejo del trauma esplénico ha cambiado de manera evidente ya que se han reportado más complicaciones frente a la respuesta quirúrgica, tendiendo cada día más a llevarse de manera conservadora con el fin de preservar el órgano considerando la importancia en su función inmunológica sobre todo en las primeras etapas de la vida (1), ya que como es conocido; existe un alto riesgo de compromiso inmunológico en pacientes esplenectomizados. Por otro lado, hay un contraste claro al momento de valorar el trauma esplénico en un servicio de emergencia ya que puede representar una causa de mortalidad no solamente en primer momento, sino también a mediano plazo por una ruptura tardía de hematoma subcapsular o la formación y ruptura de un pseudo aneurisma (2). Ya que el trauma o daño esplénico es por sí mismo un indicador de severidad en el trauma abdominal se expone de esta manera la necesidad de diagnosticar y estadificar de manera correcta el nivel de trauma esplénico para elegir su manejo adecuado sea este quirúrgico o conservador (3). 


\section{MÉTODO}

$\mathrm{S}$ e realizó una investigación descriptiva y documental, con revisión de la literatura actual de fuentes virtuales como el metabuscador (tripdatabase) y bases digitales, PubMed, Biblioteca Virtual de Salud (BVS) y repositorios institucionales. Los criterios de inclusión fueron publicaciones de evidencia secundaria sometida a filtro tal como revisiones sistemáticas, metaanálisis y guías de práctica clínica, para la selección de artículos se tomó en cuenta el período de los últimos cinco años, en los idiomas inglés y español según los términos MeSH: spleen, splenic rupture, trauma, abdominal injuries, therapeutics; además de los descriptores en ciencias de la salud (DeCs): abdomen, heridas y traumatismos, rotura del bazo y terapéutica.

Para el análisis se consultó el resumen del articulo y si cumplía con los criterios de inclusión se procedió a descargar el documento completo para su lectura. La búsqueda bibliográfica arrojó 46 investigaciones de las cuales se descartaron 29 por no ser pertinentes, duplicadas o sin acceso a textos completos. Finalmente existieron 17 estudios pertinentes que fueron analizados para la presente investigación y en su mayoría fueron obtenidos de la base PubMed en idioma inglés.

\section{DESARROLLO Y DISCUSIÓN}

\section{Diagnóstico}

$\mathrm{Si}$ bien una correcta anamnesis e historia clínica son herramientas fundamentales e irremplazables para orientarse al diagnóstico de traumatismo esplénico, así como identificar el mecanismo del trauma, y el sitio de contusión, muchos estudios han recopilado y analizado el rol que cumplen los diferentes estudios de imagen en el diagnóstico del trauma esplénico (4). Por un lado, se tiene al estudio FAST (Focused Abdominal Sonography for Trauma) el cual es el método de elección para pacientes hemodinámicamente inestables teniendo cerca del $98 \%$ de sensibilidad para detectar liquido intraabdominal y que termine en una laparotomía exploratoria temprana, sin embargo, tiene como desventaja el hecho de ser operador dependiente y que en ciertos pacientes puede existir dificultad al momento de realizar el examen (5), por otro lado, se ha considerado también a la ecografía convencional; sin embargo se ha determinado que el $25 \%$ de lesiones intra abdominales se pueden pasar por alto (6), por lo que no se recomienda su uso para el diagnóstico de trauma esplénico. En pacientes que se encuentran hemodinámicamente estables, la tomografía computarizada ha sido el examen de elección para el diagnóstico y evaluación de trauma esplénico, ya que tiene una sensibilidad del 97-99\% para identificar sangrado activo, daño no vascular y hematoma peri esplénico (7), lo cual contribuye al mejoramiento en la toma de decisión para un manejo conservador sobre manejo quirúrgico. $\mathrm{Y}$ aunque los criterios para intervención quirúrgica se rigen principalmente por el cuadro clínico y estado hemodinámico del paciente, los hallazgos tomográficos ayudan a corroborar la precisión del diagnóstico y han demostrado tener una reducción exitosa de laparotomías innecesarias en varios estudios (8). Sin embargo, no es un predictor confiable para la evolución del trauma esplénico, pudiendo algunos pacientes sufrir de complicaciones como un sangrado esplénico tardío o un absceso subfrénico. 


\section{Clasificación}

El trauma esplénico se lo lleva encasillando clásicamente en una clasificación creada en 1989 por la Asociación de Cirugía del Trauma (AAST) la misma que divide al trauma en cinco grados de acuerdo a la extensión del hematoma y la profundidad del daño apreciado en un estudio tomográfico el mismo que ha sido de ayuda para establecer el tipo de manejo con la posterior estadificación, lo cual abordaremos más adelante.

Tabla 1. Clasificación del trauma esplénico según la AAST.

\begin{tabular}{|c|c|c|}
\hline \multirow{2}{*}{ Grado } & \multicolumn{2}{|c|}{ Descripción de la lesión } \\
\hline & Hematoma & Subcapsular, $\leq 10 \%$ de superficie \\
\hline & Laceración & Desgarro capsular, no sangrante $\leq 1 \mathrm{~cm}$ de profundidad \\
\hline \multirow[t]{2}{*}{ II } & Hematoma & $\begin{array}{l}\text { Subcapsular, no expansivo, } 10-50 \% \text { superficie } \\
\text { intraparenquimatoso } \leq 2 \mathrm{~cm} \text {. }\end{array}$ \\
\hline & Laceración & $\begin{array}{l}\text { Desgarro capsular, hemorragia activa de } 1 \text { a } 3 \mathrm{~cm} \text { de profundidad } \\
\text { que no afecta a vasos trabeculares }\end{array}$ \\
\hline \multirow[t]{2}{*}{ III } & Hematoma & $\begin{array}{l}\text { Subcapsular, } \geq 50 \% \text { superficie o expansivo. Hematoma capsular } \\
\text { roto con hemorragia activa, hematoma intraparenquimatoso } \geq 2 \\
\mathrm{~cm} \text { o expansivo }\end{array}$ \\
\hline & Laceración & $\begin{array}{l}\geq 3 \mathrm{~cm} \text { de profundidad en el parénquima o afecta a vasos } \\
\text { trabeculares }\end{array}$ \\
\hline \multirow[t]{2}{*}{ IV } & Hematoma & Hematoma intraparenquimatoso roto sin hemorragia activa \\
\hline & Laceración & $\begin{array}{l}\text { Afecta a vasos segmentarios o hiliares, devascularizacion mayor } \\
\text { del } 25 \% \text { del bazo }\end{array}$ \\
\hline \multirow[t]{2}{*}{$\mathrm{V}$} & Hematoma & Estallido Esplénico \\
\hline & Laceración & Lesión hiliar que devasculariza al bazo \\
\hline
\end{tabular}

Tomado de American Association for the surgery of Trauma (1995).

Si bien la utilidad de dicha escala en la toma de decisiones para el manejo conservador frente al manejo quirúrgico ha sido validada en numerosos estudios de calidad, en muchos de los casos las lesiones asociadas al trauma sumado a la condición del paciente llegan a ser mandatorios en la decisión de traslado a un quirófano para resolución quirúrgica, sin siquiera tener la oportunidad de definir el grado de trauma esplénico antes de la cirugía. Por lo que la condición hemodinámica es el mejor indicador a la hora de tomar decisiones en el manejo del trauma esplénico (9).

En el año de 2017, durante el congreso mundial de cirugía de emergencia llevada a cabo en Brasil se presentó la guía de manejo del trauma esplénico de la WSES (World Society of Emergency Surgery) donde se incluye un nuevo sistema de clasificación para el trauma esplénico, el mismo que toma en cuenta a la condición del paciente y la anatomía de las lesiones (Tabla 2). 
Tabla 2. Escala de WSES.

\begin{tabular}{llll}
\hline & Clasificación WSES & ASST & Hemodinamia \\
\hline MENOR & WSES I & I-II & Estable \\
MODERADA & WSES II & III & Estable \\
SEVERA & WSES III & IV-V & Estable \\
\hline
\end{tabular}

Tomado de World Society of Emergency Surgery (2017).

La clasificación de WSES divide las lesiones del bazo en tres clases: menor (clase I), moderado (clase II y III) y grave (clase IV), ésta considera la clasificación de la AAST así como el estado hemodinámico $\mathrm{y}$ es la misma para pacientes adultos y pediátricos (2).

\section{Manejo}

Anteriormente el tratamiento de cualquier lesión del bazo se traducía en un paciente sometido a esplenectomía, sin embargo, con el avance de las técnicas y con las nuevas revisiones se ha demostrado que ésta debe evitarse especialmente si se encuentran involucrados niños, ancianos y pacientes con alguna patología hematológica, para que no se produzca una permanente susceptibilidad a infecciones bacterianas (10). Como se muestra anteriormente, la WSES sugirió agrupar las lesiones esplénicas en leves o menores, moderadas y severas. Las lesiones de bajo grado con baja frecuencia, que en la clasificación de la AAST serían grados I-III, serían leves o moderadas según sus características y se establecerá como mejor tratamiento el no quirúrgico. Sin embargo, los pacientes hemodinámicamente estables con lesiones de alto grado podrían tratarse con éxito de forma no operatoria, especialmente si se aprovechan las herramientas más avanzadas para el tratamiento de hemorragias. Por otro lado, las lesiones "menores" asociadas con inestabilidad hemodinámica a menudo deben ser intervenidas quirúrgicamente (2). Hoy se puede hablar sobre un tratamiento no invasivo que en conjunto con el monitoreo continuo de signos vitales y empleo de cristaloides efectuando el objetivo de expansor de volumen así como la transfusión de elementos sanguíneos son el tratamiento de elección frente al tratamiento quirúrgico ya que actualmente este accionar posee una tasa de éxito que supera el 90\% (11). El manejo no quirúrgico tiene como propósito preservar la función del órgano además de reducir la morbi-mortalidad relacionada con la cirugía. La embolización de la arteria esplénica ha sido un complemento eficaz como parte del tratamiento conservador para la lesión esplénica cerrada, sin embargo, las técnicas de embolización no arrojan estudios concluyentes (12). El "gold standard" del tratamiento quirúrgico es la esplenorrafia, la cual se utiliza en lesiones del bazo de grado I-II y fue descrita desde el año 1968. Esta consiste en el empleo de hemostasia y sutura directa sobre la cápsula esplénica, pero se corre el riesgo de presentar hemorragias tardías al procedimiento (6).

En cuanto al tratamiento quirúrgico existen dos opciones; la esplenectomía abierta y la esplenectomía total laparoscópica. La técnica de esplenectomía abierta consiste en realizar una incisión 
vertical en la línea media que se extiende desde la apófisis xifoides hasta la sínfisis púbica (13), que en comparación con las tres (o cuatro) pequeñas incisiones que se realizan en una esplenectomía total laparoscópica se entiende las ventajas de dicho tratamiento (14). En cuanto a técnicas de laparoscopía se describe también la esplenectomía parcial en la cual se extirpa una parte del bazo y se prefiere dejar el polo superior del órgano debido a que tiene mayor cantidad de elementos de fijación lo que evita la torsión en el posoperatorio (7). Una alternativa para reducir los riesgos de infección mencionados como consecuencia de la esplenectomía total así como la estancia hospitalaria es el autotransplante esplénico, el cual se realiza con varios cortes de bazo fijados en el epiplón sin embargo se habla de complicaciones como necrosis, anemia crónica entre otros (15). Se mencionan también varias complicaciones post-operatorias en las cuales se asocian alteraciones de la producción de inmunoglobulinas, eliminación mediada por anticuerpos $\mathrm{y}$ fagocitosis lo que por ende aumenta el riesgo de desarrollar sepsis (14). En cualquiera de las opciones que involucran el tratamiento quirúrgico se debe realizar profilaxis, ya que la extirpación parcial o total del órgano en cuestión hace al paciente susceptible a organismos como Staphylococcus aureus, Haemophilus influenzae y Streptococcus pneumoniae por lo que se debe administrar amoxicilina 500 miligramos una vez cada día o eritromicina 250 miligramos dos veces en el día, adicional a esto se menciona también el uso de cefalosporinas para el espectro contra Haemophilus influenzae.(16). La creciente prevalencia de patógenos no cubiertos por los programas de vacuna estándar (el llamado reemplazo de serotipo) puede explicar un alto riesgo en los pacientes esplenectomizados para adquirir infecciones, al igual que el deterioro inmunológico intrínseco que se encuentra en los individuos con asplenia. Por lo tanto, se recomienda que todo paciente que se haya sometido a una esplenectomía reciba una inmunización adecuada contra las bacterias encapsuladas dentro de las cuales se ecuentran vacunas para N.meningitidis, S.Pneumoniae, H. influenzae. Respecto a la administración de la vacuna para una esplenectomía programada se recomienda la administración al menos dos semanas antes de la cirugía, sin embargo, cuando se trata de una esplenectomía de emergencia donde no se ha cumplido el esquema de vacunación antes mencionado, se debe administrar las vacunas por lo menos dos semanas después de realizado el procedimiento (17). Por el contrario, dada la falta de ensayos clínicos o de datos retrospectivos adecuados, no existen indicaciones específicas sobre la necesidad de inmunizar a los individuos que se han sometido a angioembolización esplénica (14).

\section{CONCLUSIÓN}

$\mathrm{E}$ l bazo es el órgano que se lesiona con mayor facilidad dentro del trauma abdominal cerrado, cuya identificación es de vital importancia para la supervivencia del paciente traumatizado. Sin duda, establecer el diagnóstico correcto de esta patología lleva a un manejo correcto y a tiempo; reduciendo la mortalidad y morbilidad por complicaciones posteriores siendo esta la importancia de estandarizar el tratamiento y actualizar los conocimientos constantemente para un manejo oportuno del trauma esplénico.

Para el diagnóstico existen varios métodos de imagen, teniendo como pauta la estabilidad hemodinámica del paciente al momento de su abordaje inicial, si la 
hemodinamia se encuentra comprometida el método de elección es el FAST, sin embargo, si el paciente se encuentra hemodinámicamente estable se optará por una tomografía contrastada de abdomen. El estadiaje permite elegir el tratamiento y manejo adecuado del paciente, dentro de las clasificaciones más recomendadas está en primer lugar la AAST, misma que clasifica al trauma según las características de las lesiones anatómicas observadas en el estudio de imagen y como alternativa existe la clasificación WSES que engloba estas características y el estado hemodinámico del paciente y poco a poco va tomando lugar en la clasificación del trauma esplénico en mira de un correcto abordaje.

Respecto al manejo, se destaca el tratamiento conservador siendo ahora el recomendado para lesiones con estadios entre I-III, además ha sido considerado en estadios mayores con alta tasa de éxito siempre y cuando exista estabilidad hemodinámica en el paciente, la clave del manejo conservador radican en un monitoreo estricto de las constantes vitales, reposo, hidratación y analgesia para llevar de manera monitorizada y segura a una recuperación exitosa; sobre el uso de antibiótico no se recomienda de manera sistemática, por lo que debe ser individualizado para cada caso y sobre todo iniciarse si existe alteración en el estado del paciente que orienten a un síndrome de respuesta inflamatoria sistémica (SIRS). No se ha llegado a un conceso claro sobre el antibiótico a utilizarse, pero se recomienda utilizar esquemas de amplio espectro.

Además, los casos donde se recomienda el manejo intervencionista que principalmente son grados IV y $\mathrm{V}$ o cualquier grado siempre y cuando exista compromiso hemodinámico, no existe una diferencia significativa entre la utilización de técnica abierta versus laparoscópica en cuestión de mortalidad o morbilidad, sin embargo, resaltan las ventajas de la laparoscopía como el menor tiempo de recuperación con rápido retorno a actividades cotidianas y menor dolor postquirúrgico. Si es recomendable, según las diferentes literaturas revisadas, siempre y cuando sea factible la esplenectomía parcial sobre la total y sobre todo en adolescentes y niños, ya que la misma reduce las complicaciones fisiológicas posteriores respecto a la inmunodepresión secundaria a esplenectomía

\section{REFERENCIAS BIBLIOGRÁFICAS}

1. Luoto TT, Pakarinen MP, Koivusalo A. Long-term outcomes after pediatric splenectomy. Surgery. [Internet]. 2016 Jun [citado 28 de enero de 2021]; 159 (6): 1583-1590. Disponible en https://linkinghub.elsevier.com/retrieve/ pii/S0039606015010521

2. Coccolini F, Montori G, Catena F, Kluger Y, Biffl W, Moore EE, et al. Splenic trauma: WSES classification and guidelines for adult and pediatric patients. World J Emerg Surg. [Internet]. 2017 Dic [citado el 22 de enero de 2021] 12 (1): 40. Disponible en http://wjes.biomedcentral.com/articles/1 0.1186/s13017-017-0151-4

3. Petrone $P$, Anduaga Peña MF, Servide Staffolani MJ, Brathwaite C, Axelrad A, Ceballos Esparragón J. Evolution of the Treatment of Splenic Injuries: From Surgery to Non-Operative Management. Cir Esp Engl Ed. [Internet]. 2017 Oct [citado el 28 de enero de 2021];95:420-7.

4. Ghezzo AC, Adri DO, Gentile E, Yetati EL. Espectro de hallazgos por TC multidetector en el trauma esplénico y hepático. Rev Argent Radiol Argent J Radiol. [Internet]. 2018 Dic [citado el 22 de enero de 2021]. 82 (4). 168-74. Disponible en http://www.thieme- 
connect.de/DOI/DOI?10.1055/s-00381657774

5. Girard E, Abba J, Cristiano N, Siebert M, Barbois S, Létoublon C, et al. Management of splenic and pancreatic trauma. J Visc Surg. [Internet] 2016 Ago [ citado el 26 de enero de 2021];153 (4) :45-60. Disponible en:

https://linkinghub.elsevier.com/retrieve/ pii/S1878788616300194

6. Cardoso DL, Cardoso Filho FDA, Cardoso AL, Gonzaga ML, Grande AJ. Should splenic autotransplantation be considered after total splenectomy due to trauma? Rev Colégio Bras Cir [Internet]. 2018 Jul [citado 28 de enero de 2021];45. Disponible en: http://www.scielo.br/scielo.php?script=sc i_arttext\&pid=S0100-

69912018000300300\&lng=en\&tlng=en

7. Englum BR, Rothman J, Leonard S, Reiter A, Thornburg $\mathrm{C}$, Brindle $\mathrm{M}$, et al. Hematologic outcomes after total splenectomy and partial splenectomy for congenital hemolytic anemia. J Pediatr Surg. [Internet] 2016 Ene [citado 28 de enero de 2021] 51 (1): 122-7. Disponible en:

https://linkinghub.elsevier.com/retrieve/ pii/S0022346815006375

8. El-Matbouly M, Jabbour G, El-Menyar A, Peralta R, Abdelrahman H, Zarour A, et al. Blunt splenic trauma: Assessment, management and outcomes. The Surgeon. [Internet] $2016 \mathrm{Feb}$ [citado 26 de enero de 2021]; 14 (1) :52-8. Disponible en: https://linkinghub.elsevier.com/retrieve/ pii/S1479666X15000815

9. Coccolini F, Fugazzola $P$, Morganti L, Ceresoli M, Magnone S, Montori G, et al. The World Society of Emergency Surgery (WSES) spleen trauma classification: a useful tool in the management of splenic trauma. World J Emerg Surg. [Internet] 2019 Dic. [citado 28 de enero de 2021]; 14 (1): $30 . \quad$ Disponible en: https://wjes.biomedcentral.com/articles/ 10.1186/s13017-019-0246-1

10. Zarzaur BL, Rozycki GS. An update on nonoperative management of the spleen in adults. Trauma Surg Acute Care Open. [Internet] 2017 Jun [citado 28 de enero de
2021] 2 (1): 75. Disponible en: https://tsaco.bmj.com/lookup/doi/10.113 6/tsaco-2017-000075

11. Spijkerman R, Teuben MPJ, Hoosain F, Taylor LP, Hardcastle TC, Blokhuis TJ, et al. Non-operative management for penetrating splenic trauma: how far can we go to save splenic function World J Emerg Surg. [Internet] 2017 Dic. [citado 28 de enero de 2021] 12 (1): 33. Disponible en:

http://wjes.biomedcentral.com/articles/1 0.1186/s13017-017-0144-3

12. Teuben $M$, Spijkerman $R$, Pfeifer $R$, Blokhuis T, Huige J, Pape $\mathrm{H}-\mathrm{C}$, et al. Selective non-operative management for penetrating splenic trauma: a systematic review. Eur J Trauma Emerg Surg. [Internet] 2019 Dic. [citado 28 de enero de 2021] 45 (6): 979-985. Disponible en: http://link.springer.com/10.1007/s00068 -019-01117-1

13. Reinaldo LGC, Araújo Júnior RJC, Diniz TM, Moura R de D, Costa DL, Eulálio KD, et al. Recurrent kala-azar: report of two cured cases after total splenectomy. Rev Inst Med Trop São Paulo. [Internet] 2020 [citado 28 de enero de 2021] 62 (1): 31. Disponible en: http://www.scielo.br/scielo.php?script=sc i_arttext\&pid=S0036-

46652020000100506\&tlng=env

14. Huang GS, Chance EA, Hileman BM, Emerick ES, Gianetti EA. Laparoscopic Splenectomy in Hemodynamically Stable Blunt Trauma. JSLS. [Internet] 2017 [citado 28 de enero de 2021] 21 (2): 13. Disponible en: https://www.ncbi.nlm.nih.gov/pmc/articl es/PMC5444560/

15. Fransvea $P$, Costa G, Serao A, Cortese F, Balducci G, Sganga G, et al. Laparoscopic splenectomy after trauma: Who, when and how. A systematic review. J Minimal Access Surg. [Internet] 2021 ene [citado 28 de enero de 2021] 17 (2): 141-146. Disponible en: http://www.journalofmas.com/preprintar ticle.asp?id=269743; type $=0$

16. Tahir F, Ahmed J, Malik F. Postsplenectomy Sepsis: A Review of the 
Literature. Cureus [Internet]. 2020 feb [citado 28 de enero de 2021] 12 (2); Disponible en: https://www.cureus.com/articles/27673post-splenectomy-sepsis-a-review-of-theliterature

17. Buzelé R, Barbier L, Sauvanet A, Fantin B. Medical complications following splenectomy. J Visc Surg. [Internet] 2016 Ago. [citado 28 de enero de 2021] 153 (4): 277-286.

Disponible https://linkinghub.elsevier.com/retrieve/ pii/S1878788616300297

Conflicto de intereses: Los autores declaran que no existe conflicto de intereses para la publicación del presente artículo científico.

Financiamiento: Los autores corrieron con los gastos derivados del presente trabajo y declaran que no recibieron financiamiento externo.

Agradecimientos: Al Hospital General Riobamba IESS y en especial a quienes conforman el servicio de Cirugía General por su gran apoyo. 